\title{
Heartbeat: Is there any effective therapy for heart failure with preserved ejection fraction?
}

Heart failure with preserved ejection fraction (HFpEF) increasingly is recognised as a cause of disabling symptoms, yet we have little data to support medical therapy for this condition. In order to objectively assess the published data on drug therapy for HFpEF, Zheng and colleagues ${ }^{1}$ performed a systematic review and meta-analysis of 25 randomised controlled trials with a total of over 18000 patients. This analysis showed that beta-blocker therapy was associated with lower all-cause mortality compared with placebo (RR: $0.78,95 \%$ CI 0.65 to $0.94, \mathrm{P}=0.008$ ). Other studied therapies, including ACE inhibitors, aldosterone receptor blockers, and mineralocorticoid receptor antagonists were not associated with lower all-cause or cardiovascular mortality (figure 1).

In an editorial, Schnell and Donal ${ }^{2}$ discuss the diagnosis and treatment of HFpEF. There is no simple definition for this complex clinical syndrome, with considerable heterogeneity between patients included in these studies (figure 2). For example, the prevalence of prior myocardial infarction might alter the impact of beta-blocker therapy on clinical outcomes in different study populations. While much remains to be studied, they conclude "A careful evaluation of the patient, including the non-cardiovascular comorbidities, is particularly mandatory in this population in order to determine the best management and improve their symptoms, quality of life and reduce mortality." Further discussion of these issues can be found in a review article by Zakeri and Cowie $^{3}$ on HFpEF which focuses on the overall therapeutic approach and future directions (figure 3 ).

Although most cardiologists appreciate that bicuspid aortic valve (BAV) disease is associated with dilation of the aortic sinuses or ascending aorta, the association of BAV with variations in coronary artery anatomy is less well known. In a study of 84 post-mortem hearts of patients with complex congenital heart

Correspondence to Professor Catherine M Otto, Division of Cardiology, University of Washington, Seattle,WA 98195, USA; cmotto@uw.edu

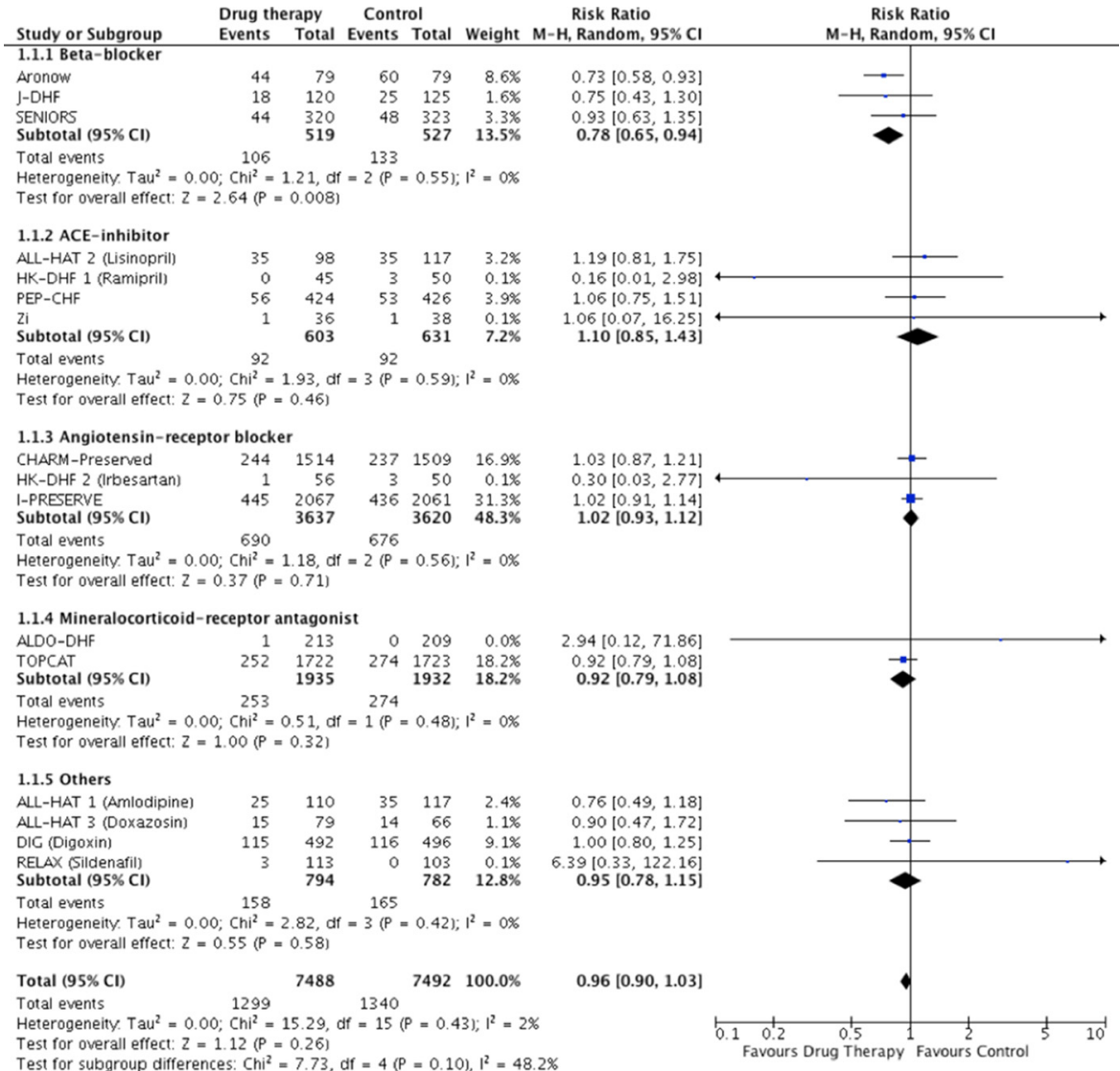

Figure 1 Pooled and individual estimates of relative risk (RR) and $95 \% \mathrm{Cl}$ of the primary outcome all-cause mortality for different therapies. Data are shown stratified by individual drug classes (beta-blockers, ACE inhibitors, angiotensin receptor blockers, mineralocorticoid receptor antagonists and other drug classes). Random-effects model used.

disease and a BAV, coronary artery variations were common with a high take-off of the right $(23 \%)$ or left $(37 \%)$ coronary artery seen most often, especially in patients with a hypoplastic left ventricle (figure 4$){ }^{4}$ As these patients grow to adulthood, general cardiologists will need to be aware of this variation in coronary anatomy.

In addition, the observations in this study may shed light on valve and coronary artery embryology, as pointed out in an accompanying editorial. ${ }^{5}$ "The genetic underpinning of abnormal coronary development is not well understood and identifying association with congenital left heart disease and aortic root abnormalities may shed some light on a potential common developmental landscape. Embryological formation of the human heart requires a complex integration of a myriad of genes, many of which are not cardiac specific, and is only partially understood. It is presumed that ectopic coronary origins occur as a consequence of disordered molecular signalling with potential genetic underpinnings."

The Education in Heart $\operatorname{article}^{6}$ in this issue summarises the incidence, pathophysiology and treatment options for coronary artery calcification with a focus on the use of rotational atherectomy in selected patients.

In the Cardiology in Focus section, Tom Treasure provides a synopsis of the early days in cardiac surgery in the UK which started by treating congenital 


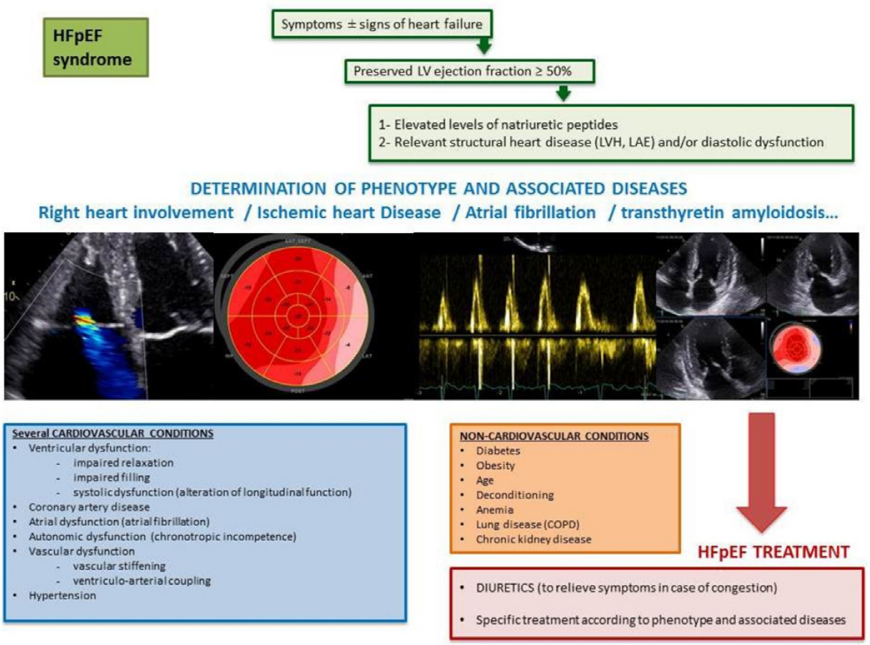

Figure 2 Management of heart failure with preserved ejection fraction (HFpEF). COPD, chronic obstructive pulmonary disease; LAE, left atrial enlargement; LV, left ventricular; LVH, left ventricular hypertrophy.

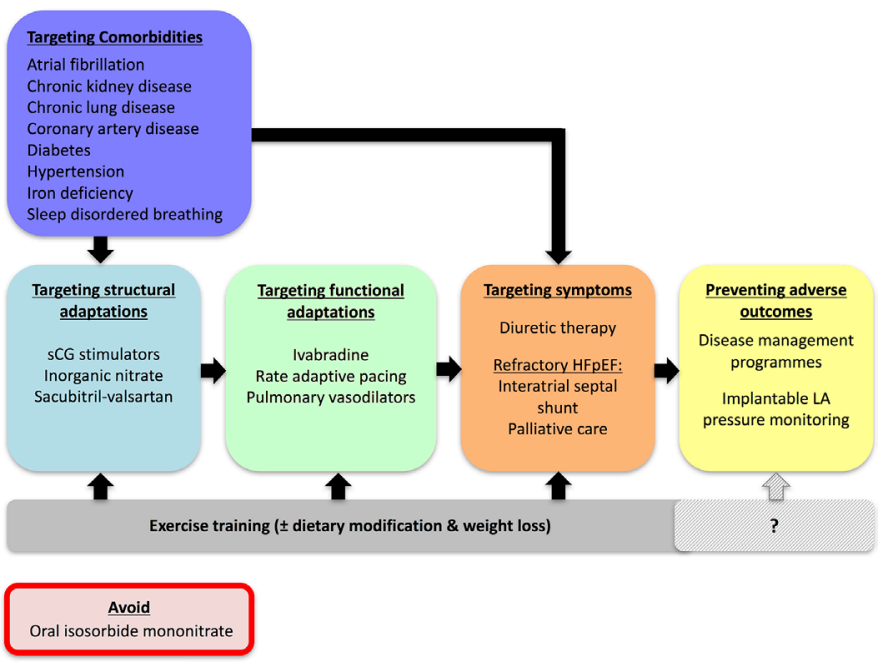

Figure 3 Therapeutic targets under investigation in HFpEF. HFpEF, heart failure with preserved ejection fraction; $\mathrm{LA}$, left atrial; $\mathrm{sCG}$, soluble guanylate cyclase.

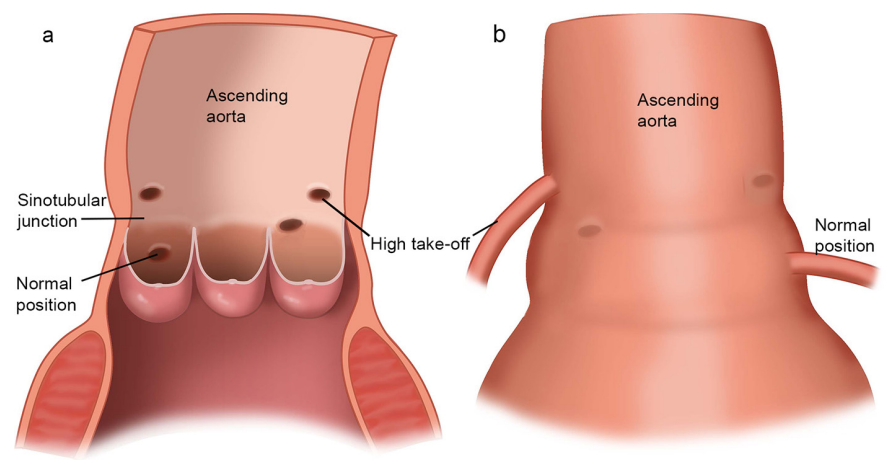

Figure 4 (a) Schematic drawing of the aortic root with the aortic sinuses. The coronary ostia can be below, on or above the sinotubular (ST) junction. Drawing shows one normal coronary ostial position, one coronary ostium on the ST junction and two coronary ostia above the ST junction. (b) Aortic root from another perspective showing one high take-off coronary artery with sharper angulation and one normal position of the coronary artery. heart disease with systemic to pulmonary shunts, followed by later development of intracardiac procedures to relieve pulmonic and mitral valve stenosis. ${ }^{7}$

The Image Challenge in this issue shows a photograph and ultrasound image of impressive right wrist swelling 4 months after coronary angiography in a 93-year-old woman being considered for transcatheter aortic valve implantation. ${ }^{8}$ How would you manage this finding?

Competing interests None declared.

Provenance and peer review Commissioned; internally peer reviewed.

(C) Article author(s) (or their employer(s) unless otherwise stated in the text of the article) 2018. All rights reserved. No commercial use is permitted unless otherwise expressly granted.

\section{Check for updates}

To cite Otto CM. Heart 2018:104:361-362.

Heart 2018:104:361-362.

doi:10.1136/heartjnl-2018-313040

\section{REFERENCES}

1 Zheng SL, Chan FT, Nabeebaccus AA, et al. Drug treatment effects on outcomes in heart failure with preserved ejection fraction: a systematic review and meta-analysis. Heart 2018;104:407-15.

2 Schnell F, Donal E. Pharmacological strategies in heart failure with preserved ejection fraction: time for an individualised treatment strategy? Heart 2018:104:365-6.

3 Zakeri R, Cowie MR. Heart failure with preserved ejection fraction: controversies, challenges and future directions. Heart 2018;104:377-84.

4 Koenraadt WMC, Bartelings MM, Bökenkamp R, et al. Coronary anatomy in children with bicuspid aortic valves and associated congenital heart disease. Heart 2018;104:385-93.

5 DeFaria Yeh D. Coronary artery variants and bicuspid aortic valve disease: gaining insight into genetic underpinnings. Heart 2018;104:363-4.

6 de Belder AJ. Rotational atherectomy: re-emergence of an old technique. Heart 2018;104:440-8.

7 Treasure T. Early days in congenital heart surgery in the UK: the Peacock Club. Heart 2018;104:438-9.

8 Maznyczka AM, Valenti D, Byrne J. Wrist mass in a 93-year-old woman. Heart 2018;104:437. 